\title{
¿Qué es la terapia colaborativa?
}

\author{
Alexis Ibarra Martínez \\ Universitat Autònoma de Barcelona \\ alexisaim@hotmail.com
}

Mi intención en este escrito es situar en contexto y describir un tipo de práctica terapéutica. Se trata de la aproximación colaborativa propuesta por Harlene Anderson y Harold Goolishian.

Bajo el rubro de "psicoterapia" se agrupan una infinidad de formas de pensamiento y acción, que pueden ser tan parecidas o tan opuestas entre sí, dependiendo de quién entra en ese territorio y con qué propósito.

En consecuencia, el tipo de descripción que se puede formular de la terapia en general, y de la aproximación colaborativa en particular, está circunscrita al contexto de reflexión en que se localiza.

La reflexión que hago en las siguientes líneas parte de una distinción entre psicología académica y psicología profesional. La primera se ocupa de la formación de teoría y de la investigación, la segunda está dirigida a una práctica (Polkinghorne, 1992).

La psicoterapia pertenece a una vertiente práctica de la psicología.

Aquí voy a hablar del enfoque colaborativo como un conjunto de formas de pensar y hacer que surgieron en la práctica, en el proceso de responder a circunstancias y demandas específicas.

\section{Antecedentes}

La raíz de la terapia colaborativa está en el movimiento sistémico o interaccional, al que comúnmente se denomina "terapia familiar".

La expresión terapia familiar es imprecisa, designa una unidad de tratamiento, mientras que la visión interaccional modificó la forma de conceptualizar el papel del terapeuta, el proceso de la terapia y las quejas de los pacientes.

La visión sistémica constituye como su objeto de reflexión e intervención a las relaciones interpersonales. Surge así la posibilidad de considerar los "problemas psicológicos" en contexto, no como manifestaciones de propiedades internas, si no como parte de pautas de interacción (Anderson, 1999; Hoffman, 1981; Hoffman, 2002;).

Las nociones de contexto y relaciones permitieron la transición hacia otros marcos que también reflexionaban en torno a ellas, principalmente el construccionismo social y la crítica postmoderna. 


\section{El escenario}

Aquí regreso a la idea de psicoterapia como práctica para hacer algunos apuntes. El punto de partida del recuento anterior es un marco conceptual, el sistémico, el punto de llegada otro marco conceptual: el construccionismo ${ }^{1}$.

Podría parecer que el desplazamiento de un punto a otro se pensó y se originó en un plano puramente teórico, no fue así. La terapia colaborativa no surgió de una idea a priori de que la terapia tendría que ser postmoderna.

El catalizador del cambio fueron las tensiones entre la experiencia del hacer y los medios disponibles para dar cuenta de esa experiencia (Anderson, 1997).

En ese sentido puede ser útil dar una imagen del escenario en que se daba la terapia. Mi objetivo es ilustrar la forma en que los cambios en dicho escenario dieron origen a prácticas alternativas. Prácticas que posteriormente se anidarían en el construccionismo y la crítica postmoderna.

Las terapias de corte interaccional fueron las primeras en reunir familias en el consultorio. Paralelamente, los espejos unidireccionales se convirtieron en un elemento intrínseco a la práctica.

Ambos elementos convirtieron las relaciones interpersonales en procesos observables y modificables. Al mismo tiempo, el espejo permitió que el trabajo clínico se realizara en equipos, compuestos por el terapeuta y los observadores detrás del espejo (Hoffman, 1981, Hoffman, 2002).

Si bien las familias eran entidades visibles para el terapeuta, el equipo y la lógica de sus intervenciones eran invisibles para el paciente.

El equipo y el espejo fueron presencias incuestionables, hasta que un suceso fortuito alteró este acomodo. Se trataba de uno de esos casos "imposibles", en el que se había intentado "todo".

La solución fue aparentemente simple, se decidió cambiar la luz y el sonido de habitación, para que la familia y su terapeuta pudieran escuchar el intercambio entre los miembros del equipo que estaban detrás del espejo.

Este acomodo azaroso es ahora una práctica habitual del conjunto de terapias construccionistas y postmodernas. Se trata del equipo reflexivo (Andersen, 1987; Andersen, 1991; Andersen, 1995).

El nuevo arreglo altera la lógica implícita que organiza a muchos tipos de terapia. Además, encarna los ideales de las terapias construccionistas.

La terapia se abre a un espacio público, dando cabida al escrutinio. El paciente no recibe un producto terminado, si no que es testigo de un intercambio y escucha distintas opiniones.

El vocabulario y la actitud del equipo cambian, ya no hablan detrás de la familia, si no frente a ella. Aparece un lenguaje cotidiano y la jerga profesional se translada al margen.

El habla y la escucha aparecen como posiciones que organizan el intercambio. El paciente transita de una posición de habla a una de escucha.

\footnotetext{
${ }^{1}$ Lo que no implica que haya una relación de continuidad entre ambos marcos.
} 
Se da lugar a la multiplicidad. Cada miembro presenta un punto de vista único, el objetivo es intercambiar opiniones, no alcanzar consenso.

La figura del experto se diluye. Ya no hay un juicio único que dicta cómo es o cómo debería actuar el paciente. Hay visiones diferentes ancladas a un contexto local de producción.

Las cualidades propias del equipo de reflexión han sido descritas aquí como un efecto de la modificación del escenario físico. En el siguiente apartado retomo dichos cualidades para situarlas en las premisas que hoy nutren la propuesta colaborativa.

\section{Una postura filosófica}

La terapia puede describirse como un hacer, que no ocurre en un vacío teórico o social, por el contrario el hacer se hace inteligible dentro de un marco de pensamiento. Cada marco constituye un mundo particular de acción y existencia que crea terapeutas diferentes que ven a personas diferentes por razones diferentes (Goolishian y Anderson, 1992).

¿Qué tipo de acciones caracterizan a la terapia colaborativa? ¿Cuál es el marco que les da visibilidad?

Este enfoque sostiene que el conocimiento, el lenguaje y las relaciones interpersonales son ámbitos inseparables. El conocimiento es relacional (se crea y se transforma en el intercambio social) y el lenguaje es generativo (conforma nuestras vidas y relaciones) (Anderson, 1997).

Estas premisas se expresan en una analogía: la terapia cómo conversación. La terapia es una relación que se da en y a través del lenguaje. Esta analogía señala cuál es el papel del terapeuta, las características del proceso y a los miembros del sistema terapéutico.

Para el terapeuta colaborativo, un "problema" no es una entidad objetiva y localizable. Por el contrario, hay tantas definiciones de problema como conversaciones en torno a él.

Los integrantes del sistema terapéutico son las personas que están en conversación sobre el problema. Ellos definen las cuestiones relevantes para el intercambio, en la medida en que las descripciones de la queja se modifican, también cambian los integrantes de dicho sistema. ${ }^{2}$

En una versión burda, un terapeuta es un participante más dentro de esta red conversacional. No obstante, su participación no está exenta de intencionalidad o responsabilidad.

El tipo de participación del terapeuta se ha descrito como una postura filosófica: una forma de ser en relación con el otro, es también una posición de reflexión sobre los conocimientos y sesgos del terapeuta (Anderson, 1997, Anderson, 2003b).

La aproximación colaborativa se interroga sobre la influencia del conocimiento en las relaciones interpersonales y viceversa. Tradicionalmente, el terapeuta es visto como el poseedor un conocimiento, conocimiento que determina su relación con el paciente.

${ }^{2}$ Luego, la terapia que delimita a priori la unidad de tratamiento (individuo, pareja, familia) carece de sentido. 
El terapeuta que adopta esta postura crea una relación que abre espacio para la construcción de conocimiento, de nuevos significados. Con este afán, muestra una actitud de respeto, curiosidad y apertura; hace públicos sus supuestos, al mismo tiempo que asume su carácter contingente y relativo.

Desde esta postura filosófica, el terapeuta participa en una exploración compartida. El intercambio toma forma a partir del vocabulario, las descripciones y narraciones del paciente ${ }^{3}$ (Anderson, 1997; Levin, London y Tarragona, 1998).

El proceso terapéutico se acerca a las características de la conversación cotidiana.

Aquí vuelvo a la idea de la terapia como conversación, vale la pena recordar que se propone como una analogía, es decir, se construyen semejanzas posibles entre dos cosas que inicialmente son diferentes.

No se trata por lo tanto de ignorar las diferencias obvias entre ambos tipos de intercambio. El potencial de la analogía radica en la apertura de rutas de acción y relación.

Al pensar en conversaciones cotidianas, el habla y la escucha aparecen como elementos que dan forma a la relación.

La escucha del terapeuta suele considerarse de carácter informativo, va encaminada a desvelar un estado de cosas. Las expresiones del terapeuta revelan al paciente la naturaleza real del problema y dictan las acciones pertinentes (Anderson, 1997, Anderson, 2003a).

En contraste, esta aproximación propone que la escucha y el habla son una forma de acción conjunta.

Las expresiones del terapeuta son una continuación de su escucha, van encaminadas a la comprensión y el reconocimiento del otro, son una invitación a que amplíe su relato (Anderson, 1997, Anderson, 2003).

La comprensión no es un proceso finito, es parte de la construcción conjunta de significados, como tal es generativa, da lugar a nuevas descripciones y narraciones, abre posibilidades de cambio.

El bosquejo de las líneas anteriores pretende dar una visión general de la práctica colaborativa y las premisas de que se nutre.

Puede resultar difícil visualizar este tipo de terapia, ya que no se estructura a partir de acciones, técnicas o pasos específicos. El reto para el practicante es traducir esta postura para responder a las particularidades de la situación. Luego, las expresiones y acciones del terapeuta varían de acuerdo al paciente y al contexto.

${ }^{3}$ Esta idea tiene una implicación doble: crea un contexto para que las personas se describan en sus propios términos y además los ubica como arquitectos de su proceso terapéutico. 


\section{Movimientos relacionados.}

La aproximación colaborativa no representa un movimiento aislado, existen otras prácticas terapéuticas que se inspiran en el pensamiento postmoderno, me refiero en concreto al enfoque centrado en soluciones y al enfoque narrativo. ${ }^{4}$

Los tres enfoques comparten premisas, que no siempre se traducen en formas de hacer similares. Al mismo tiempo, tienen concepciones encontradas del proceso y del papel del terapeuta.

Las tres orientaciones critican la noción de una realidad objetiva, una definición representacionista del lenguaje y la idea de un self autónomo y observable (Anderson, 2003b).

Transladar esta crítica a la psicoterapia implica un cuestionamiento del vocabulario diagnóstico y de la psicopatología, en particular de los efectos que tienen sobre las vidas de las personas. Así mismo se critica el tipo de relación que se instaura entre los poseedores de este vocabulario y quienes reciben sus etiquetas (Gergen, Hoffman \& Anderson, 1995).

En el ámbito de la propuesta, los tres enfoques subrayan el papel formativo del lenguaje y las relaciones interpersonales (Anderson, 2003b).

La terapia no se piensa como esfera cerrada al contexto social, si no como un espacio público. Esta premisa tiene distintas ramificaciones.

En primer lugar, el terapeuta se ubica en una posición de responsabilidad abierta, en la que da cuenta de los sesgos que informan su trabajo y acciones ${ }^{5}$.

En segundo lugar, se cuestiona el papel de la psicoterapia en la reproducción de la cultura dominante y los efectos de opresión que puede ejercer.

En respuesta a este riesgo, los tres enfoques proponen acciones para descentralizar la figura del terapeuta.

De igual manera, la terapia se propone como un espacio para fomentar un sentido de comunidad y dar voz a los saberes de la red social del paciente (Hoffman, 2002).

Si bien las ideas anteriores son en mayor o menor grado compartidas, cada enfoque hace traducciones diferentes de ellas. En las siguientes líneas, hago un bosquejo de los rasgos propios de la terapia centrada en soluciones y de la terapia narrativa.

El enfoque centrado en soluciones traza una distinción entre dos juegos de lenguaje opuestos: el habla sobre problemas y el habla sobre las soluciones, dicho en otras palabras, no es necesario hablar del problema y sus causas para construir el cambio (Berg \& de Shazer, 1993; de Shazer, 1993; Miller \& de Shazer, 1998).

\footnotetext{
${ }^{4}$ En este apartado hablaré únicamente de las terapias que tienen como antecedente al movimiento interaccional o sistémico.

${ }^{5}$ La palabra inglesa accountability expresa esta posición que quiero describir.
} 
En el encuentro terapéutico se genera una visión de la vida del paciente sin el problema. Las intervenciones del terapeuta están encaminadas a encontrar recursos y fortalezas en las personas y a identificar excepciones, es decir, aquellos eventos u ocasiones en que la queja está ausente.

Esta práctica sostiene una visión no-patologizante de las personas y sus dificultades. El terapeuta parte de un conjunto de herramientas específicas, en ese sentido asume una postura estratégica en la producción del cambio. Sin embargo no se plantea como una autoridad que dicta qué tipo de vida debe tener el paciente.

Por otra parte, la terapia narrativa adopta una analogía del texto: nuestras vidas y relaciones se constituyen a través de relatos (Freedman y Combs, 1996; White, 1995; White \& Epston, 1990).

La posibilidad de narrar está constreñida por los discursos que circulan en la cultura. Estos discursos moldean la experiencia, la identidad y las relaciones. Así el sufrimiento individual no está separado del aspecto político.

Esta aproximación parte de una distinción ente experiencia vivida y narrada. La narración nunca puede fijar en su totalidad la experiencia, esto permite separarse de y resistir a los discursos dominantes.

El terapeuta y el paciente se involucran en la construcción conjunta de relatos alternativos, que coinciden con las maneras preferidas de vivir y relacionarse de la persona.

Este enfoque propone un conjunto de acciones específicas, el elemento distintivo es la conversación externalizante, que sitúa al problema por fuera del individuo. Esta práctica contradice el supuesto de que los problemas están dentro de las personas.

De esta forma el paciente puede reflexionar sobre su relación con el problema e identificar aquellos acontecimientos en que ha logrado vencerlo o resistirlo.

Otra práctica representativa es el uso de documentos escritos como medio de resistencia al peso que tienen los expedientes psiquiátricos.

\section{Reflexiones finales}

Junto con el equipo de reflexión, las terapias colaborativa, narrativa y de soluciones conforman el paisaje construccionista de la práctica clínica.

Estas aproximaciones no se proponen como sistemas teóricos, si no como invitaciones a pautas de acción y relación (de ahí la distinción entre psicología académica y práctica).

Tampoco se estructuran a partir de un arsenal de técnicas, que el terapeuta aplica mecánicamente. La terapia no es un proceso instrumental y unidireccional que consiste en hacer a, si no en hacer con el otro.

Quien entiende la práctica terapéutica en términos instrumentales, o en términos de un sistema teórico niega que sucede en contextos concretos y por tanto únicos. La práctica es por definición ambigua y no es conocible desde una posición de exterioridad (Schön, 1983; Shotter \& Katz, 1996).

Por ello, entiendo las propuestas anteriores como formas de ver y pensar que sirven al terapeuta para orientarse y responder a situaciones específicas. 
En mi opinión, la relación que estas aproximaciones sugieren ocupa un primer plano. En ese sentido es importante tener herramientas conceptuales que den cuenta de la cualidad interpersonal de la terapia, que al mismo tiempo permitan explorar los potenciales y limitaciones del contexto terapéutico.

Por otra parte, parece haber una contradicción inherente a la expresión "psicoterapia postmoderna", en tanto que la terapia es hija directa de la modernidad. El reto, por tanto, está en que las prácticas aquí descritas puedan salir de las cuatro paredes del consultorio.

El primer paso ya está dado, estos enfoques tienden a incluir a la red social de las personas. También reúnen a individuos no-profesionales que participan como testigos de la experiencia del otro y como expertos en determinadas situaciones de vida.

Dentro de estas prácticas hay un marcado interés por las formas de intercambio que hacen viable el diálogo y colaboración. Algunas de estos formatos para el diálogo se han llevado a ámbitos extraterapéuticos.

Así hay intervenciones que se plantean desde un inicio como comunitarias. Por ejemplo, el trabajo conocido como "diálogos abiertos" incluye a toda la red social de personas etiquetadas como psicóticas, así como al sistema de profesionales. El acento se coloca en generar redes de intercambio y en las formas de habla de los participantes, no en la eliminación de los síntomas (Hoffman, 2002).

Las formas de conversación que son útiles en psicoterapia se han llevado al ámbito de confrontaciones o controversias públicas, por ejemplo, las oposiciones entre grupos pro-vida y proelección (Chasin, Herzig, Roth, Chasin, Becker \& Stains, 1996).

Así, la psicoterapia emerge como un espacio para explorar el papel del habla y la escucha en la generación del diálogo.

\section{Referencias}

Andersen, T. (1987). The reflecting team: Dialogue and meta-dialogue in clinical work. Family Process, 26(4), 415-428.

Andersen, T. (1991). El equipo reflexivo: Diálogos y diálogos sobre los diálogos. Barcelona: Gedisa.

Andersen, T. (1995). Reflecting processes, acts of informing and forming: you can borrow my eyes, but you must not take them away from me! En S. Friedman (Ed.), The reflecting team in action: Collaborative practice in family therapy. New York: Guilford Press.

Anderson, H. (1997). Conversation, language and possibilities: A postmodern approach to therapy. Nueva York: Basic Books.

Anderson, H. (1999). Reimagining family therapy: reflections on Minuchin's invisible family. Journal of Marital and Family Therapy, 25, 1-8.

Anderson, H. (2003a). Listening, hearing and speaking: thoughts on the relationship to dialogue. En: www.harlene.org/writings/ 
Anderson, H. (2003b). Postmodern social construction therapies. En: www.harlene.org/writings/

Berg, I. K. \& de Shazer, S. (1993). Making numbers talk: language in therapy. En: Friedman, S. (Ed). The new language of change. Nueva York: The Guilford Press.

Chasin, R., Herzig, M., Roth, S., Chasin, L., Becker. C. \& Stains, R. (1996). From diatribe to dialogue on divisive public issues: approaches drawn from family therapy. En: http://www.publicconversations.org/pcp/resources/resource detail.asp?ref id=61

Freedman, J. \& Combs, G. (1996). Narrative therapy: The social construction of preferred realities. New York: Norton.

Gergen, K. J., Hoffman, L. \& Anderson, H. (1995). Is diagnosis a disaster: a constructionist trialogue. En:

http://www.swarthmore.edu/SocSci/kgergen1/web/page.phtml?id=manuscripts\&st=manuscri pts

Goolishian, H. \& Anderson, H. (1992). Strategy and intervention versus nonintervention: a matter of theory? Journal of Marital and Family Therapy, 18: 5-15.

Hoffman, L. (1981). Foundations of family therapy. Nueva York: Basic Books.

Hoffman, L. (2002). Family therapy: An intimate history. Nueva York: Norton.

Levin, S., London, S., \& Tarragona, M. (1998). Hearing more voices: beyond traditions in writing, research, and therapy. Journal of Systemic Therapies, 17(4): 1-87.

Miller, G. \& de Shazer, S. (1998). Have you heard the latest rumor about...? Solution-focused therapy as a rumour. Family Process, 37: 367-377.

Polkinghorne, D. E. (1992). Postmodern epistemology of practice. En: Kvale, S. Psychology and postmodernism. Londres: Sage.

Schön, D. (1983). The reflective practitioner: How professionals think in action. Nueva York: Basic books.

Shazer, S. de (1993). Creative misunderstanding: there is no scape from language. En: Gilligan, S. \& Price, R. (Eds) Therapeutic conversations. Nueva York: Norton.

Shotter, J. y Katz, A. M. (1996). Articulating a practice from within the practice itself: Establishing formative dialogues by the use of a social poetics. Concepts and transformations, 1, 213237. Disponible en: http://pubpages.unh.edu/ ids/page2.htm

White, M. (1995). Reescribir la vida: entrevistas y ensayos. Barcelona: Gedisa.

White, M. \& Epston, D. (1990). Medios narrativos para fines terapéuticos. Barcelona: Paidós.

\section{Formato de citación}

Ibarra, A. (2004). ¿Qué es la terapia colaborativa? Athenea Digital, 5. Disponible en http://antalya.uab.es/athenea/num5/ibarra.pdf 